\title{
RAPID ACQUISITION OF ENVIRONMENTAL INFORMATION AFTER ACCIDENTS WITH HAZARDOUS CARGO THROUGH REMOTE SENSING BY UAV
}

\author{
G. A. Longhitano ${ }^{\text {a }}$, J. A. Quintanilha ${ }^{\mathrm{b}}$ \\ ${ }^{\text {a }}$ PROCAM - University of São Paulo - georgelonghitano@yahoo.com.br \\ ${ }^{\mathrm{b}} \mathrm{EP}$ - University of São Paulo - jaquinta@ usp.br
}

KEY WORDS: Remote Sensing, Environmental Impacts, Highway Transportation, Hazardous Cargoes

\begin{abstract}
:
The aim of the study was to present procedures of using remote sensing by UAV to aid in the rapid evaluation and monitoring of environmental impacts caused by accidents involving transport of dangerous cargoes on highways. From the data collected and the UAV model defined, it was developed a methodology of operating a UAV to generate the potential benefits identified in the application. A Mini UAV or Close Range class was considered appropriate. It was created the procedures for imaging, the emergency activation of an UAV, the generation parameters of the images and forms of environmental information extraction. The study does not eliminate the necessity of testing the technology for this application, but indicates the possibility of generating more detailed and faster information.
\end{abstract}

\section{INTRODUCTION}

The transport of dangerous products is performed in Brazil, about $70 \%$, by road (Alves et al., 2009).

Only in the state of São Paulo, $40.5 \%$ of environmental accidents treated between 1978 and 2008 by Technology Company of Environmental Sanitation of the State of São Paulo (CETESB, 2009), was caused by highway transportation of dangerous loads. The average number of this kind of accidents recorded from 2004 to 2008, was 216 per year in the state of São Paulo.

Accidents involving transport of hazardous materials can cause different environmental impacts, such as contamination of soil, surface and groundwater, impacts to wildlife and vegetation, damage to public and private property and economic activities such as agriculture. They may even cause damage to the health and welfare of man.

Because of this, there must be an environmental impact assessment for planning actions to control, mitigation and compensation of impacts, and environmental monitoring of recovery of affected areas.

The affected areas and objects (soils, vegetation, water, for example) must be identified and the direct and indirect environmental impacts evaluated. It should also be performed environmental diagnosis of the accident site and its surroundings to assist the management and planning of actions and anticipated new impacts or even the dynamic of the identified impacts. The information generated can also be used for legal prosecution of environmental violations to those responsible for transport.

The identification, quantification and monitoring of environmental impacts caused by these kind of accidents represent a challenge, because there is a wide variety of scenarios for the care of an emergency, which carries risks and difficulties for response teams.

In environmental studies, remote sensing is recognized as a very useful tool. An alternative relatively recent is through the use of UAVs, which are able to obtain images with very high spatial and temporal resolutions at the same time, what is indicated for disaster management, as outlined in Jensen (2007) and in Argrow \& Frew (2009).

Aerial images obtained from areas affected by accidents with dangerous cargo, since that done quickly, could help teams to remotely assess the environmental situation faster and safely and with the possibility of generating better information. On the other hand, there are technique questions about the on the ability of these systems sensors acquire images of the affected areas in a timely manner with the desired quality and without prohibitive costs.

It's also important consider that, although there is an increase in the number of projects aimed at implementing civil UAVs (ISPRS, 2011), this can still be considered incipient, particularly in the Brazilian scenario.

Performed this considerations, the aim of the study was to present procedures of using remote sensing UAV to aid in a rapid evaluation and monitoring of environmental impacts caused by accidents involving transport of dangerous cargoes on highways.

\section{METHOD}

Besides literature search performed on the subjects it covers the research, it was conducted some interviews and questionnaires were administered with Brazilian UAVs companies, with technical sector of chemical emergency response by CETESB and with specialized environmental consulting companies.

Thus, a large number of hypothetical environmental scenarios that could occur after accidents involving transportation of hazardous materials were identified.

Considering the variety of scenarios identified, and the possibilities and capabilities of UAVs, were collected the benefits that technology can bring concerning usual technical procedures.

Procedures that may benefit in speed, security, and increase quality and efficiency through the use of images of the surface obtained by remote sensors carried by UAVs were identified. Knowing the specific circumstances and the assessment of environmental impacts in an emergency, the embedded platform and sensor system for aerial imaging, in case a UAV, must 
combine ease and speed of operation and at the same time, imaging with spatial and spectral resolution allowing identification and quantification of affected areas.

The main desired capabilities of remote sensing in areas affected by any disaster is providing images that combine high temporal resolution (revisit time of a few hours or less) with high spatial resolution (sub-meter preferably), to evaluate the impacts occurred and planning response actions (MEULEMAN et al., 2007).

The best parameters and the most appropriate capabilities of a UAV were defined and it was developed a methodology of operating a UAV to the required use. Throughout the process considerations were conducted evaluating the advantages and technical limitations of the use of technology.

Finally, it was studied the Brazilian laws for the use of UAVs to verify the legal feasibility.

\section{RESULTS}

\subsection{Environmental Scenarios after Accident with Hazardous Cargo on Roads}

Most of the environmental impacts caused by an accident occurs immediately, at events, with leakage of the load. The substance that seeps in contact with air, soil, water, plants, animals and humans, may cause a lot of damage immediately, within moments, minutes, or even in a matter of hours after the accident. Therefore, these impacts are classified as short-term occurrence. Medium-term impacts to occur may also exist, but are rarer. Examples include contamination of aquifers, where it is needed the percolation of substances in the soil and contamination and indirect killing of specimens of fauna through the food chain.

About the scale of these impacts, it is necessary to estimate the size of the areas that are usually affected by accidents involving with road transport of dangerous goods for parameterization of the size of areas that an image must cover. In this sense, it was researched on the scale of the phenomenon, which refers to the size of the geographical phenomenon's manifestation on the earth's surface.

Based on information from CETESB, organ featuring teams that carry out impact assessments on accidents involving the transport of dangerous cargo, the affected areas typically range from tens of square meters to $10.000 \mathrm{~m}^{2}$. In general, the areas directly affected have a few hundred square meters. The volumes of substances that can be discharged vary, but it can be considered that a tanker truck currently carries up to $50 \mathrm{~m}^{3}$ volumes.

It should be distinguished that the manifestation of these impacts may vary between punctual, linear and areal geographical space. The relief influence is important.

When the spilled product reaches a surface drainage, the area of contamination may extend linearly for over kilometres, with product shipping by river flow. It should be noted that extensions and areas affected by accidents involving carriage of dangerous cargoes may be larger than those taken as references, although in rare cases.

Besides the affected areas, should also be characterized the areas surrounding these to predict impacts of neighbourhood, and if the impact will continue occurring or even if new impacts can be generated.

Determining the type of soil of the site and whether water bodies where the substance is leaking is important to knowing the magnitude of impact and to determine control measures.
Given these circumstances, a diagnosis must be made around the affected areas. The areas to be raised in these surroundings, vary, but usually no more than $300 \mathrm{~m}$ away.

In extreme cases can reach linear distances of the order of kilometres, when there is river transport of substances spilled.

Some examples of direct environmental impacts that can be generated through accidents involving transportation of hazardous materials on highways are: soil contamination, surface water contamination, groundwater contamination, contamination of humans, terrestrial fauna contamination, aquatic fauna contamination, contamination and death of vegetation, burning of vegetation, loss of agricultural activities and loss of public or private property. There is also the possibility of indirect environmental impacts, such as stoppage of water supply of a city, due to contamination from surface water sources or groundwater, for example.

\subsection{Characteristics of a UAV for the Application}

A Mini UAV class or Close Range was considered appropriate to the application. It must have specific characteristics like for example: range of up to $8 \mathrm{~km}$, flight range of at least 30 minutes, flying height of $2000 \mathrm{~m}$, to be able to take off and land in small areas and quickly.

It also recommends the ability to fly day and night and mostly, the capacity to operate under adverse weather conditions such as rain and moderate winds. The UAV must also have a level of Safety according to its characteristics.

It is recommended that the UAV present ability to perform missions programmed or autonomous, with possibility of programming of geographic referenced points of acquisition.

The information and images in real time transmission function, primarily through video, is recommended to be displayed important environmental elements, which can also determine the imaging of specific areas previously to the landing of UAV generating ability to obtain relevant images. The storage of information is essential for cases of transmission failure.

The UAV systems to be used must be able to reach the place of occurrence and obtain images very quickly, along with the care team, and must have agile deployment and operation to provide answers soon. After reaching a place for takeoff, deployment, operation and imaging by the system, it should not take more than half hour.

Trained professionals should always be available for UAV system operation, image acquisition, processing and analysis of them.

Regarding the sensor to be embedded, it is recommended that it generates colour images at least of the visible region, although multispectral sensor information with near infrared bands would be useful to identify more accurately environmental damage to vegetation and affected areas.

Other sensors options should not be discarded for the application, but this analysis does not show at first a costeffective technical and economic justification for the application in analysis.

It is noteworthy that regardless of these options, the highest spatial resolution of the images that usually UAVs are able to generate should allow the identification of phenomena and objects on the surface more easily. Rocchini (2007), for example, demonstrated the best efficiency of both spectral and spatial resolutions, in studies of vegetation to compare the use of data from different sensors.

Within Brazilians UAVs currently operating, there are models which can serve for the desired application. The UAV AGplane, from AGX Company was used as reference in this study. 
The aircraft weighs $20 \mathrm{~kg}$ and measures 3,2 $\mathrm{m}$ wingspan and 2,2 $\mathrm{m}$ long, has a capacity of up to $10 \mathrm{~kg}$ payload, flight endurance of $3,5 \mathrm{~h}$ and range of $8 \mathrm{~km}$. It reaches heights of up to $3 \mathrm{~km}$. It can be classified as a Close Range UAV. It takes off coupled by car, which is the same as its transportation and land in areas short of a few meters, or even via parachute assisted manner. The imaging and flight plan can be performed autonomously. The aircraft can be equipped with conventional digital cameras (cameras currently with 10 to 21 megapixels) or other sensors. The company also has a sensor AGX multispectral capable of imaging regions of the visible and near infrared (NIR) that can be shipped.

The aircraft provides geographic referenced images automatically, with accuracy of GPS navigation (approximately $15 \mathrm{~m})$, transmits real-time videos and images to ground control station and can fly under conditions of rain and moderate winds and overnight. A ground pilot and an assistant / reserve pilot are sufficient for operation.

\subsection{Procedures to use remote sensing by UAV to aid in the rapid evaluation and monitoring of environmental impacts}

The feasibility of full implementation would depend on an organization and ready intelligence, linked to routine methodology and service teams, and linked to the flowchart of the steps of the highways Emergency Plans Attendances.

The procedures determined by the laws and regulations that apply to the state of São Paulo were used as references. In other countries and states, the emergency response generally follows similar procedures, but there are cases where there are specific laws and standards of care for these cases accidents with dangerous cargo transportation.

Once activated, the professional equipped with the UAV should immediately depart for obtaining aerial imagery. Obtaining images should occur as soon as possible and preferably within half hour.

The determination of the area to be imaged depends on the characteristics of the area and the accident. Initially, a standard covering area around $300 \mathrm{~m}$ of the exact spot of the accident can be obtained.

The areas may be increased according to the conditions of the local topography, weather (rain) and transport of materials by water in rivers, lakes or reservoirs. Still, in case of fire generated by the accident, where fire can spread, the affected areas may be considerably bigger.

It should be noted that weather conditions may impair the acquisition of aerial imagery and depending on the intensity of winds, rain and snow, the UAV may have restrictions on flying. Obtaining data overnight, also complicates, requiring NIR sensors and better trained professionals to image interpretation. The aerial images generated must have some characteristics as: multispectral, GSD better than $0,5 \mathrm{~m}$ and geographic referenced with $15 \mathrm{~m}$ accuracy.

If required immediately a mapping, it is possible to delimit affected areas through photointerpretation with manual drawing polygons over the image, or also accomplish a digital classification quickly if the geometric accuracy is neglected.

As environmental techniques will be near the affected area, visible elements in the same field in the images may be associated.

Thus information can be obtained from basic orientation and location of elements identified only in the image, even if it is not geographic referenced.

A good photo interpreter with aerial image can locate and extract environmental information with the aerial image in minutes. In the application studied, it is more important to generate fast thematic date, with no need to accurately quantify the areas.

An example of image from UAV AGplane and a digital classification by segmentation with Brazilian free software Spring is showed at Figure 1.

Thus, the extent of these contaminated areas and, in addition to soil type, topography, vegetation, hydrograph, land use and proximity to sensitive receptors points can be extracted from the images.

This information will support the identification and evaluation of environmental impacts and vulnerability of the areas, as well as the adoption of immediate actions to control and mitigate the impacts. After, the data can be used for documentation of accident.
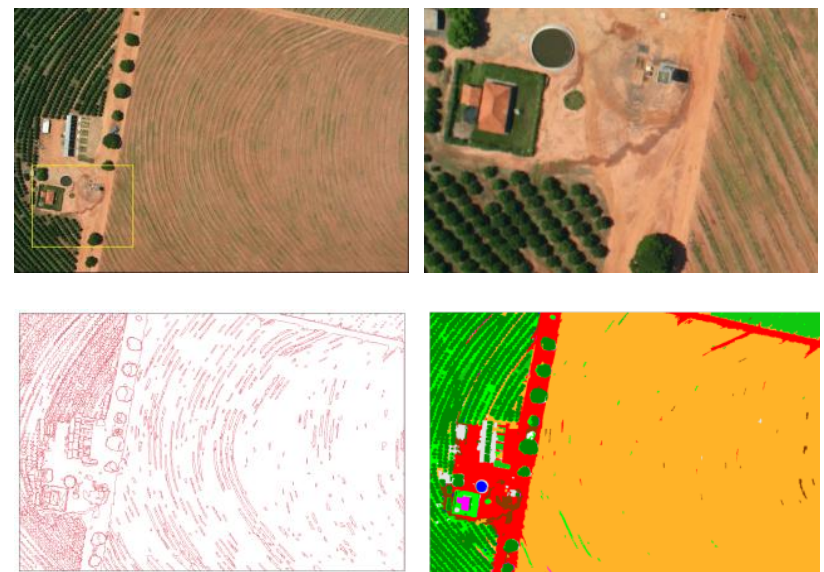

Figure 1. Sample image (GSD of $0,14 \mathrm{~m}$ ) obtained with AGplane and digital classification.

Through these image processing shows up, as a corollary, that using images taken by drones, spilled liquids on the ground can be identified, classified and mapped. These patterns are similar to identified impacts that can be generated by accidents with dangerous cargo transportation.

However, it should be noted that there may be cases where the images would not show good efficacy. There are substances that are extremely volatile and evaporate very fast, leaving no surface traces observable. There is also possibility of disposal of substances to vegetated areas that have closed canopy, preventing the display of the affected areas through aerial images.

Technical efficiency will obviously depend on the environmental setting of the area of the accident and characteristics of the cargo, and the obtaining of remote sensing images of affected areas does not replace the need for environmental impact assessment by field technicians. It should assist rather with the possibility of generating more, and in faster and safer way.

Aerial images generated to evaluate the occurrence and immediate monitoring can assist in generating information for a number of documents required for registration and description of the environmental character of the occurrence.

Not only images can be used but also visual representations or cartographic obtained through these would be useful.

Once on this stage, the information need not to be generated so quickly, more lengthy procedures can be made in order to obtain more accurate information and better representations of impacts and environmental characteristics of the areas.

To place a mapping through images is necessary to identify, division and grouping (classification) of classes corresponding to the existing surface. For this there are different methods and 
techniques, analog or digital, unsupervised or supervised procedures.

From the data collected in the field in the attendance of the event, through research on secondary data and job office, where remote sensing images performed by UAVs can be used to obtain and consolidate environmental information, as demonstrated, can be performed a qualitative assessment of environmental impacts.

The magnitude of impact and resilience of the environment are associated with many factors such as the type and quantity of the product involved, the physical characteristics of the environment and biological communities hit.

For each of the identified impacts, the assessment should be carried out using criteria, which should assist in these qualitative measurement and determination of management actions that must be taken for each one of them. Considering the potential impacts of accidents involving the transport of dangerous goods, some criteria can be applied as follows: reversibility, coverage, relevance, magnitude, duration, occurrence, incidence and time of occurrence.

Using the criteria listed, the company responsible for the report, or CETESB, can assess the significance of environmental impacts caused by the accident with clarity, which should also subsidize the choice and decision by the possible measures for monitoring, remediation and rehabilitation of the area, in addition to compensation measures of impacts.

Normally the description of the affected and surrounding areas is performed only by means of text, sometimes accompanied by pictures and illustrative sketches. Aerial photographs would enhance and facilitate this description.

As for the environmental impact assessment, aerial images serve to document the subsequent management actions and could be used in the composition of monitoring reports from the adoption of management actions.

The aerial images could be used in this way, to obtain an evolutionary framework of the impacted area and surroundings, considering the adoption of management actions.

\subsection{Considerations About Brazilian Laws to Use UAVs}

By cohabit the airspace used by manned aircraft and overflying populated areas, the UAVs activity should be regulated, since it can cause serious damage and risks to aviation and population. Guidelines and rules for legal certification of activity are in discussion in several locations around the world.

In Brazil, there are rules, but still underdeveloped. The DCEA Department of Brazilian Airspace Control presented the first paper that provides and regulates activity in the country (AIC) $29 / 09$ of $19 / 11 / 2009$, which aims to present the information necessary for the use of UAVs in the Brazilian airspace and recognizes the application of technology in Brazil.

This circular sets provisionally, while there is no more specific legislation and regulation to that operation. To operate an UAVs in Brazil, it is required a authorization document permit to be issued by the organs of Civil Aviation and Military.

UAVs for civilian use in Brazil, have not been allowed on flights over populated areas. Moreover, to obtain authorization, the delay can be more than 30 days after the submission to ANAC - National Agency of Civil Aviation. Exceptions can only be made to public safety agencies, such as police.

This legal framework, which is highly restrictive in many applications of this technology, such as in emergency situations, can only be changed when generated a more complete resolution regulating the activity of UAVs in Brazil.

\section{CONCLUSIONS}

It is correct to say that there are, nowadays, UAVs that have desirable parameters for emergency applications and are technically capable of generating images quickly to evaluate environmental impacts post-accident with dangerous cargo transportation. But the research does not eliminate the requirement for tests and the proposed procedures don't replace the necessity for technical work in the field. It assists with generation of more detailed, safer and faster information.

While there may be variations of some parameters to be involved and detailed feasibility studies should be performed for each specific use case, the UAV can be applied in a similar manner on an emergency basis after other types of environmental disasters of similar spatial extent $\left(<100.000 \mathrm{~m}^{2}\right)$ as in landslides or floods, or to accidents with other modes of transportation of hazardous cargo, such as railroads and pipelines.

It is understood that the information generated can serve including allowance for future research and usage of UAVs for remote sensing in various emergency situations.

In relation to the Brazilian laws, these possibilities of applications in rapid acquisition of environmental information currently suffer with the way of obtaining flight to UAVs, which just forbids the speed and flexibility of these new remote sensing platforms.

It is up to regulatory bodies create a more complete and less restrictive statute, while ensuring the safety of the airspace and flown over the area and allow the full potential of development and applications that the UAVs technology presents. Brazil, with its continental dimensions and physical characteristics, coupled with the enormous lack of geographical data, presents a great potential for UAV applications, an activity that is growing notoriously.

\section{REFERENCES}

Alves, P. et al. 2009. Análise do transporte rodoviário de produtos perigosos no estado de São Paulo e suas consequências no meio ambiente. In: Anais do VI Congresso de Meio Ambiente da AUGM. São Carlos, Brazil.

Argrow, B., W., E., \& Frew, E. W., 2009. "Real-Time Participant Feedback from the Symposium for Civilian Applications of Unmanned Aircraft Systems," Journal of Intelligent and Robotic Systems, Vol. 54, No. 1-3, pp. 87-103.

CETESB - Companhia de Tecnologia e Saneamento Ambiental. 2009. Relatório de estatísticas de atendimentos a acidentes ambientais. São Paulo.

IEEE, 2009. Transactions on Geoscience and Remote Sensing, vol 47, n.3.

ISPRS, 2011. International Conference on Unmanned Aerial Vehicle in Geomatics (UAV-g), Volume XXXVIII-1/C22. Zurich, Switzerland.

Jensen, J. R., 2007. Remote Sensing of the Environment: An Earth Resource Perspective, 2nd Ed. Prentice Hall, Upper Saddle River, NJ.

Meuleman, K. et al. 2007. Seveseo: eo-based services in support to industrial and technological risk management. In: Proc. 'Envisat Symposium 2007, 23-27. Montreux. 
International Archives of the Photogrammetry, Remote Sensing and Spatial Information Sciences, Volume XL-1/W1, ISPRS Hannover Workshop 2013, 21 - 24 May 2013, Hannover, Germany

Rocchini, D., 2007. Effects of spatial and spectral resolution in estimating ecosystem $\alpha$-diversity by satellite imagery. Remote Sensing of Environment, v. 111, p. 423-434,. 